\title{
Ultrareliable Short-Packet Communications With Wireless Energy Transfer
}

\author{
Onel L. Alcaraz López, Hirley Alves, Richard Demo Souza, and Evelio Martín García Fernández
}

\begin{abstract}
We analyze and optimize a wireless system with energy transfer in the downlink and information transfer in the uplink, under quasi-static Nakagami-m fading. We consider ultrareliable communication scenarios representative of the fifth generation of wireless systems, with strict error and latency requirements. The error probability and delay are investigated, and an approximation for the former is given and validated through simulations. The numerical results demonstrate that there are optimum numbers of channels uses for both energy and information transfer for a given message length.
\end{abstract}

Index Terms-.

\section{INTRODUCTION}

W IRELESS energy transfer (WET) is emerging as a potential technology for powering small and energy-efficient devices with low-power requirements through radio frequency (RF) signals [1]. This comes with the implicit advantage that RF signals can carry both energy and information [2]. This scenario becomes very attractive for future communication paradigms such as the Internet of Things, where powering a potentially massive number of devices will be a major challenge [3].

As pointed out in [4], the most important characteristics of WET systems are the following:

1) power consumption of the nodes is in the order of microwatts;

2) strict requirements on the reliability of the energy supply and of the data transfer;

3 ) information is conveyed in short packets.

This third requirement is due to intrinsically small data payloads, low-latency requirements, and/or lack of energy resources to support longer transmissions [5]. Indeed, short packets are essential to support ultrareliable communication (URC) [6], which is a novel operation mode under discussion for the fifth generation $(5 \mathrm{G})$ of wireless systems. URC over a short term (URC-S)

This work was supported by CNPq, CAPES, Fundação Araucária (Brazil) and Academy of Finland, and the Program for Graduate Students from Cooperation Agreements (PEC-PG, of CAPES/CNPq Brazil). The associate editor coordinating the review of this manuscript and approving it for publication was Dr. Luca Rugini. (Corresponding author: R. D. Souza.)

O. L. A. López and E. M. G. Fernández are with the Federal University of Paraná, Curitiba 80060-000, Brazil (e-mail: onel.luis@ufpr.br; evelio@ufpr.br).

$\mathrm{H}$. Alves is with the Centre for Wireless Communications, University of Oulu, 90014 Oulu, Finland (e-mail: halves@ee.oulu.fi).

R. D. Souza is with the Federal University of Technology Paraná, 80230-901 Curitiba, Brazil (e-mail: richard@utfpr.edu.br). focuses on how to deliver short packets under stringent latency $(\leq 10 \mathrm{~ms})$ and error probability (e.g., $10^{-5}$ ) requirements [7].

Although performance metrics like Shannon capacity, and its extension to nonergodic channels, have been proven useful to design current wireless systems, they are no longer appropriate in a short-packet scenario [7]. This is because such performance metrics are asymptotic in the packet length and fit well for systems without hard delay requirements [8]. The Shannon capacity implies that an arbitrarily low-error probability can be achieved when sufficiently long packets are used, i.e., introducing sufficiently long delays. In the case of short packets, a more suitable metric is the maximum achievable rate at a given block length and error probability. This metric has been characterized in [9] and [10] for both additive white Gaussian noise (AWGN) and fading channels. In addition, the authors of [11] and [12] incorporate the cost of acquiring instantaneous channel state information (CSI) within a transmission deadline and analyze the impact of a target error probability for different scenarios under a finite block-length regime.

WET systems with short packets have been recently investigated in the literature. In [13], subblock energy-constrained codes are investigated, and a sufficient condition on the subblock length to avoid energy outage at the receiver is provided. In [5], a node, charged by a power beacon, attempts to communicate with a receiver over a noisy channel. The system performance is investigated as a function of the number of channel uses for WET and for wireless information transfer (WIT). An amplify-and-forward relaying scenario is analyzed in [14], and tight approximations for the outage probability/throughput are given. Retransmission protocols, in both energy and information transmission phases, are implemented in [4] to reduce the outage probability compared to open-loop communications. Optimal power allocation and time sharing between the energy and information signals is proposed to minimize the energy-constrained outage probability.

Differently from the above works, this letter aims at energyconstrained URC-S scenarios with both error probability and latency constraints. The system is composed of a point-topoint communication link under Nakagami-m quasi-static fading, with WET in the downlink and WIT in the uplink. The main contributions are as follows: 1 ) we derive a closed-form approximation for the WIT error probability, as a function of the amount of channel uses in the WET and WIT phases, and validate its accuracy through simulations; and 2) we show the existence of optimum values for the amount of channel uses in the WIT and WET phases, and that by increasing the WIT block length, the required WET block length for a given target reliability decreases. Our results also show that the more stringent the 


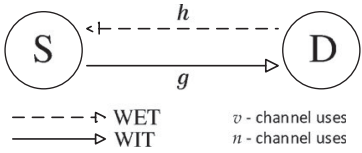

Fig. 1. System model with WET in the downlink and WIT in the uplink

reliability requirement, the higher the required delay, increasing also the portion of time dedicated to the WET phase. Moreover, in general, the possibility of meeting the reliability and latency constraints increases by decreasing the message length.

Next, Section II presents the system model and assumptions. Section III discusses the performance metrics and an approximation for the error probability. Section IV presents the numerical results. Finally, Section V concludes this letter.

Notation: $X \sim \Gamma(m, 1 / m)$ is a normalized gamma distributed random variable with shape factor $m$ and probability density function (PDF) $f_{X}(x)=\frac{m^{m}}{\Gamma(m)} x^{m-1} e^{-m x}$. Let $\mathbb{E}[\cdot]$ denote expectation, while ${ }_{x} F_{y}$ and $\mathrm{K}_{t}(\cdot)$ are the generalized hypergeometric function and the modified Bessel function of second kind and order $t$, respectively [15].

\section{SYSTEM MODEL}

Consider the scenario in Fig. 1, in which $S$ represents the information source, while $D$ is the destination. The nodes are single antenna, half-duplex, devices. $D$ is assumed to be externally powered and acts as an interrogator, requesting information from $S$, which may be seen as a sensor node with very limited energy supply. First, $D$ charges $S$ during $v$ channel uses in the WET phase, through channel $h$. Then, $S$ uses the energy obtained in the WET phase to transmit $k$ information bits over $n$ channel uses in the WIT phase ${ }^{1}$ through channel $g$. The duration of a channel use is denoted by $T_{c}$. Nakagami-m quasi-static channels are assumed, where the fading process is considered to be constant over the transmission of a block (either of $v$ or $n$ channel uses) and independent and identically distributed from block to block. We consider independent normalized channel gains; then, $h^{2} \sim \Gamma(m, 1 / m)$ and $g^{2} \sim \Gamma(m, 1 / m)$. In addition, perfect CSI at $D$ is assumed in the decoding after the WIT phase. ${ }^{2}$

\section{A. WET Phase}

In this phase, $D$ charges $S$ during $v$ channel uses. The energy harvested at $S$ is given by

$$
E=\frac{\eta P_{D} h^{2}}{\kappa d^{\alpha}} v T_{c}
$$

where $P_{D}$ is the transmit power of $D, 0<\eta<1$ is the energy conversion efficiency, $d$ is the distance between $S$ and $D, \alpha$ is the path loss exponent, and $\kappa$ accounts for other factors as

\footnotetext{
${ }^{1}$ Different (and possibly very distant) frequency bands for both processes are assumed, motivated by the different properties of the WET and WIT reception/transmission circuits as in [4] and [16]-[18].

${ }^{2} \mathrm{CSI}$ acquisition in an energy-limited setup is not trivial and including the effect of imperfect CSI would demand a more elaborated mathematical analysis, which is out of the scope of this work. However, notice that when channels remain constant over multiple transmission rounds, the cost of CSI acquisition can be negligible. Anyway, our analysis based on perfect CSI gives an upperbound on the performance of real scenarios.
}

the carrier frequency, heights and gains of the antennas [19]. In addition, we assume that $P_{D}$ is sufficiently large such that the energy harvested from noise is negligible.

\section{B. WIT Phase}

After the WET phase, $S$ uses the harvested energy to transmit a message of $k$ bits to $D$ over $n$ channel uses. The signal received at $D$ can be written as

$$
y_{D}=\sqrt{\frac{P_{S}}{\kappa d^{\alpha}}} g x_{S}+w_{D}
$$

where $x_{S}$ is the zero-mean unit-variance Gaussian codebook transmitted by $S, \mathbb{E}\left[\left|x_{S}\right|^{2}\right]=1, w_{D}$ is the Gaussian noise vector at $D$ with variance $\sigma_{D}^{2}$, and the transmit power is

$$
P_{S}=\frac{E}{n T_{c}}=\frac{\eta v P_{D} h^{2}}{n \kappa d^{\alpha}} .
$$

Thus, the instantaneous signal-to-noise ratio at $D$ is

$$
\gamma=\frac{\eta v P_{D} h^{2} g^{2}}{n \kappa^{2} d^{2 \alpha} \sigma_{D}^{2}}=\frac{\eta v P_{D} \tilde{h} \tilde{g}}{m^{2} n \kappa^{2} d^{2 \alpha} \sigma_{D}^{2}}=\mu z
$$

where $\tilde{u}=m \cdot u^{2}, u \in\{h, g\}$, is distributed according to the standard gamma PDF, $\mu=\frac{\eta v P_{D}}{m^{2} n \kappa^{2} d^{2 \alpha} \sigma_{D}^{2}}$, and $z=\tilde{h} \tilde{g}$, whose PDF is given by [20, Th. 2.1]

$$
f_{Z}(z)=\frac{2}{\Gamma(m)^{2}} z^{m-1} \mathrm{~K}_{0}(2 \sqrt{z}), \quad z>0 .
$$

\section{Performance AT Finite Block Length}

We consider a time-constrained setup, which implies that $D$ has to decode the received signal block by block. The system performance is characterized in terms of error probability and delay when $S$ is transmitting at a fixed rate $r=k / n$.

\section{A. Error Probability and Delay}

Let $\epsilon$ be the average error probability, which, for quasi-static fading channels and sufficiently large values of $n$, e.g., $n \geq 100$, can be approximated as [10, eq. (59)]

$$
\epsilon \approx \mathbb{E}\left[Q\left(\frac{C(\gamma)-r}{\sqrt{V(\gamma) / n}}\right)\right]=\int_{0}^{\infty} Q\left(\frac{C(\mu z)-r}{\sqrt{V(\mu z) / n}}\right) f_{Z}(z) d z
$$

where $C(\gamma)=\log _{2}(1+\gamma)$ is the Shannon capacity, $V(\gamma)=$ $\left(1-\frac{1}{(1+\gamma)^{2}}\right)\left(\log _{2} e\right)^{2}$ is the channel dispersion, which measures the stochastic variability of the channel relative to a deterministic channel with the same capacity [9], and $Q(x)=$ $\int_{x}^{\infty} \frac{1}{\sqrt{2 \pi}} e^{-t^{2} / 2} d t$. Let $\delta$ be the delay in delivering a message of $k$ bits, while $\delta^{*}$ is the minimum delay that satisfies a given reliability constraint. Moreover, $\nu$ is the time-sharing parameter representing the fraction of $\delta$ devoted to WET only. Both metrics are given next

$$
\begin{aligned}
\delta & =n+v \\
\nu & =v / \delta .
\end{aligned}
$$

Notice that $\delta$ is measured in channel uses, while $\delta T_{c}$ would be the delay in seconds. Finally, we define the optimum WIT block 
length, in the sense of minimizing $\delta^{*}$, as $n^{*}$. Both $\delta^{*}$ and $n^{*}$ are numerically investigated in Section IV.

\section{B. Error Probability Approximation}

It seems intractable to find a closed-form solution for (6). Then, first, we resort to an approximation of $Q(p(\mu z)), p(\mu z)=$ $\frac{C(\mu z)-r}{\sqrt{V(\mu z) / n}}$, given by [4], [21]

$$
Q(p(\mu z)) \approx \Omega(\mu z)= \begin{cases}1, & z \leq \zeta^{2} \\ \frac{1}{2}-\frac{\beta}{\sqrt{2 \pi}}(\mu z-\theta), & \zeta^{2}<z<\xi^{2}(9) \\ 0, & z \geq \xi^{2}\end{cases}
$$

where $\zeta^{2}=\frac{\varrho}{\mu}, \xi^{2}=\frac{\vartheta}{\mu}, \quad \theta=2^{k / n}-1, \quad \beta=\sqrt{\frac{n}{2 \pi}}\left(2^{2 k / n}-\right.$ 1) $)^{-\frac{1}{2}}, \varrho=\theta-\frac{1}{\beta} \sqrt{\frac{\pi}{2}}$, and $\vartheta=\theta+\frac{1}{\beta} \sqrt{\frac{\pi}{2}}$, which leads to the following result.

Theorem 1: For the system described in Section II, the error probability in (6) can be approximated as in (10).

$$
\begin{aligned}
& \mathbb{E}[\epsilon] \approx \zeta^{2 m} \frac{\left(\omega_{1}-\omega_{3}\right)}{m}\left[\mathrm{~K}_{0}(2 \zeta){ }_{1} F_{2}\left(1 ; m, m+1 ; \zeta^{2}\right)\right. \\
& \left.+\frac{\zeta}{m} \mathrm{~K}_{1}(2 \zeta){ }_{1} F_{2}\left(1 ; m+1, m+1 ; \zeta^{2}\right)\right]+\zeta^{2 m+2} \frac{\omega_{2}}{m+1} \\
& \times\left[\mathrm{K}_{0}(2 \zeta) \cdot{ }_{1} F_{2}\left(1 ; m+1, m+2, \zeta^{2}\right)+\frac{\zeta}{m+1} \mathrm{~K}_{1}(2 \zeta)\right. \\
& \left.\times{ }_{1} F_{2}\left(1 ; m+2, m+2, \zeta^{2}\right)\right]+\xi^{2 m} \mathrm{~K}_{0}(2 \xi) \\
& \times\left[\frac{\omega_{3}}{m}{ }_{1} F_{2}\left(1 ; m, m+1 ; \xi^{2}\right)+\right. \\
& \left.-\frac{\omega_{2} \xi^{2}}{m+1}{ }_{1} F_{2}\left(1 ; m+1, m+2 ; \xi^{2}\right)\right]+\xi^{2 m+1} \mathrm{~K}_{1}(2 \xi) \\
& \times\left[\frac{\omega_{3}}{m^{2}}{ }_{1} F_{2}\left(1 ; m+1, m+1 ; \xi^{2}\right)\right. \\
& \left.-\frac{\omega_{2} \xi^{2}}{(m+1)^{2}}{ }_{1} F_{2}\left(1 ; m+2, m+2 ; \xi^{2}\right)\right]
\end{aligned}
$$

Proof: See the Appendix.

\section{NUMERICAL RESUlTS}

In the numerical results, we consider scenarios with stringent error probability requirements, which are expected to be typical of URC-S services in future $5 \mathrm{G}$ systems. Therefore, being $\epsilon_{0}$ the target error probability, $\epsilon \leq \epsilon_{0}$ must be satisfied. Results are obtained by setting $m=3, \alpha=3$, and $d=12 \mathrm{~m}$. We also assume that $\kappa=10^{3}$, which is equivalent to $30-\mathrm{dB}$ average signal power attenuation at a reference distance of $1 \mathrm{~m}$. Following the state of the art in the circuit design, we consider $\eta=0.5$ [22]. Moreover, $P_{D}=30 \mathrm{dBm}$ and $T_{c}=3 \mu \mathrm{s}$; thus, $\sigma_{D}^{2}=-110 \mathrm{dBm}$ is a valid assumption if a bandwidth of around $1 \mathrm{MHz}$ is assumed.

Fig. 2 shows $\delta^{*}$, and the corresponding $\nu$, when messages of $k=216$ bits are transmitted with $100 \leq n \leq 5000$ channel uses. Particularly, the top of Fig. 2(a) shows the minimum required delay $\delta^{*}$, while the corresponding time-sharing parameter $\nu$ is plotted at the bottom of Fig. 2(b). We can note that there is an optimum value for the WIT block length, $n^{*}$, which increases as $\epsilon_{0}$ decreases but always keeps relatively small $\left(n^{*}<500\right.$ channel uses) and, at the same time, the optimum WET time increases. As shown in Fig. 2(b), when $n$ increases, the portion of time devoted for WET decreases and the optimum WET block length remains very small for $\epsilon_{0}=10^{-3}$. When $\epsilon_{0}=10^{-5}$ (reliability of $99.999 \%$ ), the minimum allowable delay is about

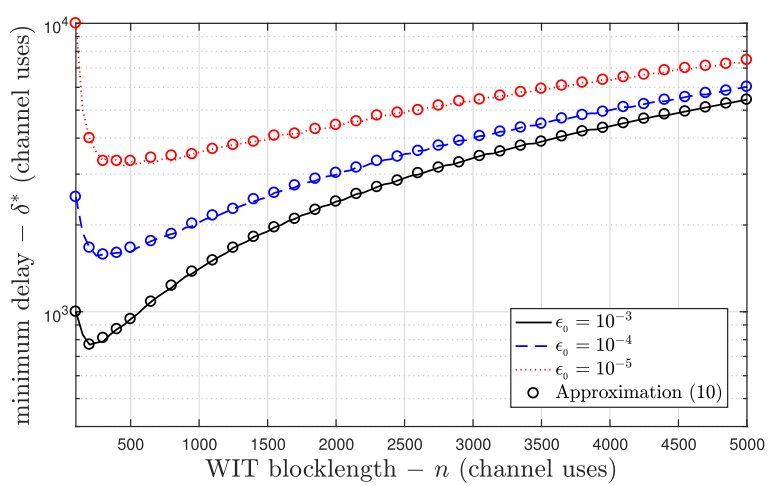

(a)

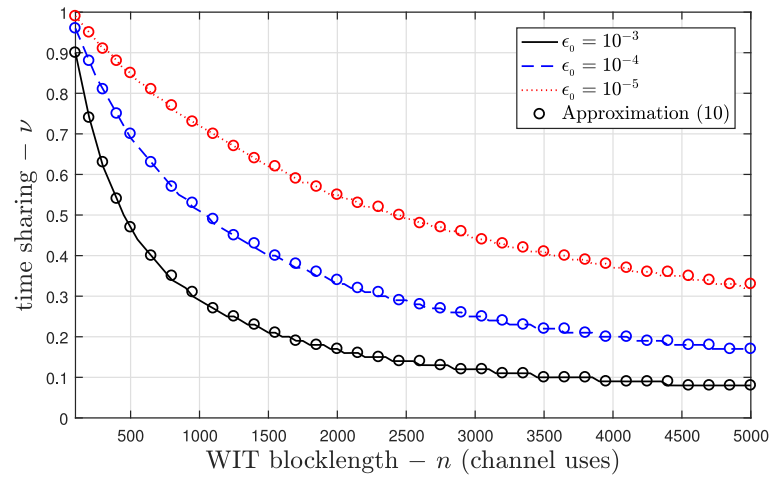

(b)

Fig. 2. (a) $\delta^{*}$ (top) and (b) $\nu$ (bottom), for packets of $k=216$ bits, as a function of the WIT block length $n$.

3300 channel uses $\sim 10 \mathrm{~ms}$, due to the large value of the required WET block length, which could be very severe for some URC-S scenarios. Moreover, it is interesting to note that the approximation derived in (10) agrees very well with the numerical integration of (6), which is accurate for $n>100$ for AWGN [9, Figs. 12 and 13] and fading channels [10].

Retransmission protocols can be very convenient to improve the system performance as investigated in [4] and [21] for other scenarios. In that sense, an error probability of $10^{-6}$ can be achieved via $\epsilon_{0}=10^{-3}$ by allowing for just one packet retransmission and causing a delay much smaller than 3000 channel uses. However, such performance improvement would only be possible if the retransmissions are subject to independent channel realizations (i.e., if the channel coherence time is not larger than the block length). In our work, we aim at showing the performance in a simple open-loop scenario, where the channel coherence time is larger than the block length. On the other hand, and as shown in Fig. 3, reducing the message length $k$ helps in improving the minimum required delay for a given $\epsilon_{0}$, which is also in line with previous results for a system without delay restrictions [4, Fig. 8]. For instance, if $k=128$ information bits, then $\delta^{*} \approx 2000$ channel uses $\sim 6 \mathrm{~ms}$, which meets more stringent system requirements. For a given reliability requirement, and based on (6), when $k$ increases, $n$ should increase as well in order to diminish the rate $r$; hence, $\gamma$ and $C(\gamma)$ tend to decrease. Also, increasing $v$ slows the capacity decrease and the required $n$ does not need to be so large; thus, a tradeoff between increasing $v$ and $n$ is identified. Nevertheless, increasing $k$ with a fixed $\epsilon_{0}$ leads to an inevitable increase in the overall number of channel uses $n+v$, which is also clearly inferred from Fig. 3 . 


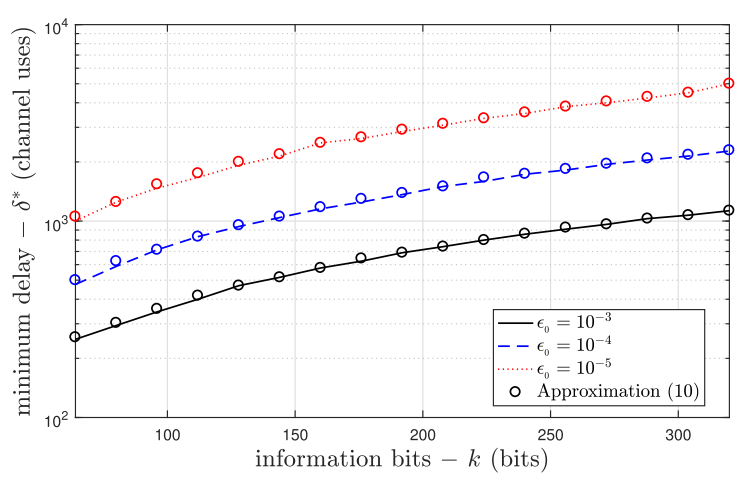

Fig. 3. $\delta^{*}$ as a function of message length $k$ for different $\epsilon_{0}$.

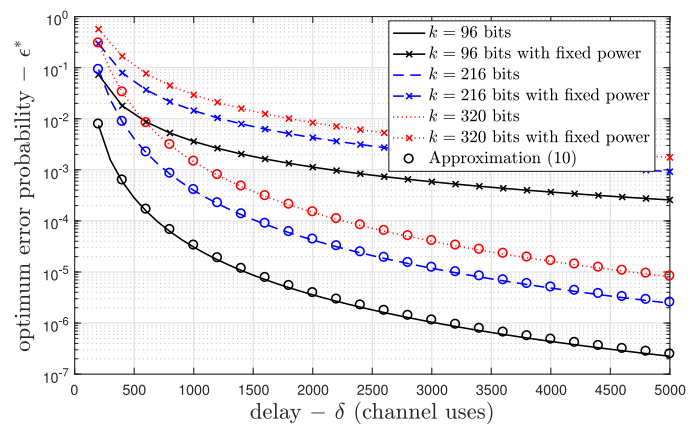

(a)

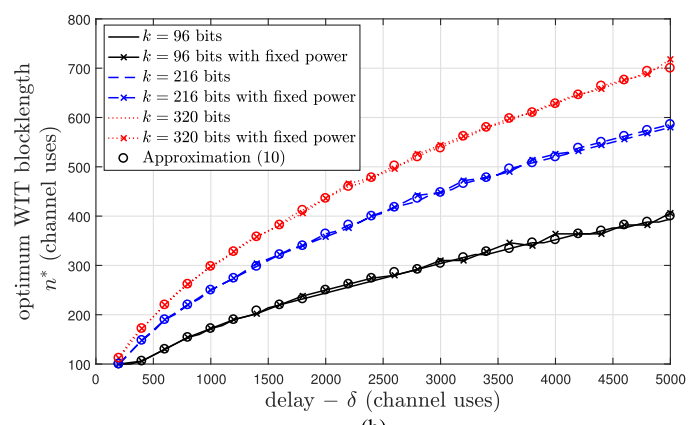

(b)

Fig. 4. (a) $\epsilon^{*}$ (top) and (b) $n^{*}$ (bottom), for packets of $k=96, k=216$, and $k=320$ bits, as a function of the delay.

Finally, in Fig. 4(a), we evaluate the minimum achievable error probability $\epsilon$ for different delays $\delta$, and we also compare the system performance with that of a scenario, where node $S$ transmits with the optimum fixed power $\hat{P}_{S}^{*}$. Notice that in such a scenario, an outage can also occur when the harvested energy is insufficient for $S$ to transmit with power $\hat{P}_{S}^{*}$. In addition, we do not consider energy accumulation from round to round. As expected, the performance of the fixed transmit power scheme is much worse than that with power allocation. Fig. 4(b) shows the optimum block length for WIT, $n^{*}$, which increases slowly with $\delta$, a consequence of the fact that increasing the allowed delay requires increasing more the fraction of time for WET for obtaining a smaller error probability. This means that $v$ has a greater impact on the error probability when increasing the allowable delay than the WIT block length $n$ on its own. Also, $n^{*}$ is practically the same for both scenarios.

The analysis presented in this letter was also carried out using the classical information theoretical tools that assume an infinite block length. The differences in the results when considering finite or infinite blocklenghts are small, but increase with the decrease of $k$ or with the increase of $m$, which are in line with the results reported in [23] for a scenario without WET and are omitted here for the sake of brevity. Therefore, we can conclude that the loss in performance due to the use of short packets is very small in our particular scenario.

\section{CONCLUSION}

We evaluated a communication system with WET in the downlink and WIT in the uplink, in URC-S scenarios. We provided an analytical approximation for the error probability and validated its accuracy. The numerical results demonstrated that there are optimum values for the WIT and WET block lengths. By increasing the WIT block length, the required WET block length decreases. In addition, we show that the more stringent the reliability, the higher the minimum required delay, which also increases the fraction of time devoted for WET. Finally, we show that depending on the particular requirement, WET in URC-S scenarios may be feasible.

\section{APPENDIX \\ PROOF OF THEOREM 1}

First, let us substitute (5) and (9) into (6), which yields

$$
\begin{aligned}
\mathbb{E}[\epsilon] & \approx \frac{2}{\Gamma(m)^{2}} \int_{0}^{\infty} z^{m-1} \mathrm{~K}_{0}(2 \sqrt{z}) \Omega(\mu z) d z \\
& \approx \omega_{1} \int_{0}^{\zeta^{2}} z^{m-1} \mathrm{~K}_{0}(2 \sqrt{z}) d z-\omega_{2} \int_{\zeta^{2}}^{\xi^{2}} z^{m} \mathrm{~K}_{0}(2 \sqrt{z}) d z \\
& +\omega_{3} \int_{\zeta^{2}}^{\xi^{2}} z^{m-1} \mathrm{~K}_{0}(2 \sqrt{z}) d z
\end{aligned}
$$

where $\omega_{1}=\frac{2}{\Gamma(m)^{2}}, \omega_{2}=\frac{\beta \mu}{\sqrt{2 \pi}} \omega_{1}$, and $\omega_{3}=\left(\frac{1}{2}+\frac{\beta \theta}{\sqrt{2 \pi}}\right) \omega_{1}$. In order to continue with the derivations, we need first to compute $\int z^{p} \mathrm{~K}_{0}(2 \sqrt{z}) d z$, which is given next

$$
\begin{aligned}
\int z^{p} \mathrm{~K}_{0}(2 \sqrt{z}) d z \stackrel{(a)}{=} \int\left(\frac{q}{2}\right)^{2 p+1} \mathrm{~K}_{0}(q) d q \\
\stackrel{(b)}{=}\left(\frac{q}{2}\right)^{2 p+2} \Gamma(p+1)^{2}\left[\frac{\mathrm{K}_{0}(q){ }_{1} F_{2}\left(1 ; p+1, p+2 ; \frac{q^{2}}{4}\right)}{\Gamma(p+1) \Gamma(p+2)}\right. \\
\left.\quad+\frac{\frac{q}{2} \mathrm{~K}_{1}(q)_{1} F_{2}\left(1 ; p+2, p+2 ; \frac{q^{2}}{4}\right)}{\Gamma(p+2)^{2}}\right] \\
\stackrel{(c)}{=} z^{p+1} \frac{\mathrm{K}_{0}(2 \sqrt{z}){ }_{1} F_{2}(1 ; p+1, p+2 ; z)}{p+1}+ \\
\quad+z^{p+\frac{3}{2}} \frac{\mathrm{K}_{1}(2 \sqrt{z}){ }_{1} F_{2}(1 ; p+2, p+2 ; z)}{(p+1)^{2}}
\end{aligned}
$$

where in $(a)$, we substitute $q=2 \sqrt{z}$, and (b) comes after some algebraic manipulations of the results given in [24, Sec. 1.1.2] along with the definition of ${ }_{x} F_{y}$. Finally, in $(c)$, we return to variable $z$, and substituting (12) into (11), we attain (10) given that $\lim _{z \rightarrow 0} z^{p} \mathrm{~K}_{t}(2 \sqrt{z})=0, p>\frac{1}{2}, t \in\{0,1\}$ (which comes from its series expansion at $z=0$ ), which concludes the proof. 


\section{REFERENCES}

[1] S. Ulukus et al., "Energy harvesting wireless communications: A review of recent advances," IEEE J. Sel. Areas Commun., vol. 33, no. 3, pp. 360381, Mar. 2015

[2] L. R. Varshney, "Transporting information and energy simultaneously," in Proc. IEEE Int. Symp. Inf. Theory, Jul. 2008, pp. 1612-1616.

[3] A. Zanella, N. Bui, A. Castellani, L. Vangelista, and M. Zorzi, "Internet of things for smart cities," IEEE Internet Things J., vol. 1, no. 1, pp. 22-32, Feb. 2014.

[4] B. Makki, T. Svensson, and M. Zorzi, "Wireless energy and information transmission using feedback: Infinite and finite block-length analysis," IEEE Trans. Commun., vol. 64, no. 12, pp. 5304-5318, Dec. 2016.

[5] T. A. Khan, R. W. Heath, Jr, and P. Popovski, "On wirelessly powered communications with short packets," in Proc. IEEE Globecom Workshops, Washington, DC, USA, 2016, pp. 1-6.

[6] P. Popovski, "Ultra-reliable communication in 5G wireless systems," in Proc. 1st Int. Conf. 5 G Ubiquitous Connectivity, Nov. 2014, pp. 146-151.

[7] G. Durisi, T. Koch, and P. Popovski, "Toward massive, ultrareliable, and low-latency wireless communication with short packets," Proc. IEEE, vol. 104, no. 9, pp. 1711-1726, Sep. 2016.

[8] R. Devassy, G. Durisi, P. Popovski, and E. G. Ström, "Finite-blocklength analysis of the ARQ-protocol throughput over the Gaussian collision channel," in Proc. 6th Int. Symp. Commun., Control Signal Process., May 2014, pp. 173-177.

[9] Y. Polyanskiy, H. V. Poor, and S. Verdu, "Channel coding rate in the finite blocklength regime," IEEE Trans. Inf. Theory, vol. 56, no. 5, pp. $2307-$ 2359, May 2010.

[10] W. Yang, G. Durisi, T. Koch, and Y. Polyanskiy, "Quasi-static multipleantenna fading channels at finite blocklength," IEEE Trans. Inf. Theory, vol. 60, no. 7, pp. 4232-4265, Jul. 2014

[11] S. Schiessl, H. Al-Zubaidy, M. Skoglund, and J. Gross, "Analysis of wireless communications with finite blocklength and imperfect channel knowledge," arXiv preprint arXiv:1608.08445, 2016.

[12] Y. Hu, M. Serror, K. Wehrle, and J. Gross, "Finite blocklength performance of multi-terminal wireless industrial networks," arXiv preprint arXiv:1606.08646, 2016.
[13] A. Tandon, M. Motani, and L. R. Varshney, "Subblock energy-constrained codes for simultaneous energy and information transfer," in Proc. IEEE Int. Symp. Inf. Theory, Jul. 2016, pp. 1969-1973.

[14] M. Haghifam, B. Makki, M. Nasiri-Kenari, T. Svensson, and M. Zorzi, "Wireless-powered relaying with finite block-length codes," arXiv preprint arXiv:1611.05995, 2016.

[15] I. Gradshteyn and I. M. Ryzhik, Table of Integrals, Series, and Products, A. Jeffrey and D. Zwillinger, Eds. New York, NY, USA: Academic, 2007.

[16] H. Chen, Y. Li, J. L. Rebelatto, B. F. Uchôa-Filho, and B. Vucetic, "Harvest-then-cooperate: Wireless-powered cooperative communications," IEEE Trans. Signal Process., vol. 63, no. 7, pp. 1700-1711, Apr. 2015.

[17] S. Mandal and R. Sarpeshkar, "Power-efficient impedance-modulation wireless data links for biomedical implants," IEEE Trans. Biomed. Circuits Syst., vol. 2, no. 4, pp. 301-315, Dec. 2008.

[18] G. L. Moritz, J. L. Rebelatto, R. D. Souza, B. F. Uchôa-Filho, and Y. Li, "Time-switching uplink network-coded cooperative communication with downlink energy transfer," IEEE Trans. Signal Process., vol. 62, no. 19, pp. 5009-5019, Oct. 2014

[19] A. Goldsmith, Wireless Communications. Cambridge, U.K.: Cambridge Univ. Press, 2005.

[20] C. S. Withers and S. Nadarajah, "On the product of gamma random variables," Quality Quantity, vol. 47, no. 1, pp. 545-552, 2013. [Online]. Available: http://dx.doi.org/10.1007/s11135-011-9474-5

[21] B. Makki, T. Svensson, and M. Zorzi, "Finite block-length analysis of the incremental redundancy HARQ," IEEE Wireless Commun. Lett., vol. 3, no. 5, pp. 529-532, Oct. 2014

[22] X. Lu, P. Wang, D. Niyato, D. I. Kim, and Z. Han, "Wireless networks with RF energy harvesting: A contemporary survey," IEEE Commun. Surveys Tuts., vol. 17, no. 2, pp. 757-789, Second Quarter 2015.

[23] P. Mary, J.-M. Gorce, A. Unsal, and H. V. Poor, "Finite block length information theory: What is the practical impact on wireless communications?" in Proc. IEEE Global Commun. Conf., Washington, DC, USA, Dec. 2016, pp. $1-6$.

[24] W. Rosenheinrich, "Tables of some indefinite integrals of bessel functions," University of Applied Sciences, Jena, Germany, 2012. [Online] Available: http://www.fh-jena.de/ rsh/Forschung/Stoer/besint.pdf 